\title{
q-analytic functions, fractals and generalized analytic functions
}

\author{
Oktay K Pashaev and Sengul Nalci \\ Department of Mathematics, Izmir Institute of Technology Urla-Izmir, 35430, Turkey \\ E-mail: oktaypashaev@iyte.edu.tr and sengulnalci@iyte.edu.tr
}

Received 13 November 2013

Accepted for publication 4 December 2013

Published 7 January 2014

\begin{abstract}
We introduce a new class of complex functions of complex argument which we call $q$-analytic functions. These functions satisfy $q$-Cauchy-Riemann equations and have real and imaginary parts as $q$-harmonic functions. We show that $q$-analytic functions are not the analytic functions. However some of these complex functions fall in the class of generalized analytic functions. As a main example we study the complex $q$-binomial functions and their integral representation as a solution of the D-bar problem. In terms of these functions the complex $q$-analytic fractal, satisfying the self-similar $q$-difference equation is derived. A new type of quantum states as $q$-analytic coherent states and corresponding $q$-analytic Fock-Bargmann representation are constructed. As an application, we solve quantum $q$-oscillator problem in this representation, and show that the wave functions of quantum states are given by complex $q$-binomials.
\end{abstract}

Keywords: $q$ calculus, $q$ oscillator, generalized analytic functions, FockBargman representation, fractal PACS numbers: 02.20.Uw, 02.30.Fn, 03.65.Db, 05.45.Df

(Some figures may appear in colour only in the online journal)

\section{Introduction}

'Complex variable theory is so beautiful that I feel that nature must have made good use of it, and, very likely, we need to make stronger use of it than we've done up to present' PAM Dirac

\subsection{Analytic functions in classical and quantum physics}

It is hard to overestimate the role of analytic function theory in classical and quantum physics. The main property of analytic functions as the power series expansion systematically has been used already by Newton for solving mechanical problems. D'Alembert and Euler in their study of hydrodynamic problems found the system of equations, $\varphi_{x}=\psi_{y}, \varphi_{y}=-\psi_{x}$, known 
as the Cauchy-Riemann equations, and got solution of the system as real and imaginary parts of an analytic function of complex variable [1]. For the stationary planar flow $\mathbf{v}=\left(\varphi_{x}, \varphi_{y}\right)$, this system describes incompressible $\operatorname{div} \mathbf{v}=0$ and irrotational $\operatorname{rot} \mathbf{v}=0$ fluid in terms of complex potential $f=\varphi+\mathrm{i} \psi$.

In 1851 Riemann introduced the formal complex derivatives and defined the analytic function by the holomorphicity condition $i f_{x}=f_{y}$ or $\partial f / \partial \bar{z}=0$. Later this form becomes very common in conformal geometry and analytic description of surfaces. In the 19th century, in works firstly by Cauchy, the modern theory of analytic functions has been created and developed together with problems of mathematical physics [1]. Among these, the stability criteria in vibration theory which was given by location of complex roots of analytic polynomial function. It becomes also clear that new geometry discovered by Lobachevsky is as well the geometry of analytic functions of one complex variable.

With the discovery of quantum physics, where complex wave function of the quantum states satisfies the complex amplitude addition rules for quantum probabilities, the role of complex analysis becomes even more significant. The Glauber coherent states and corresponding Fock-Bargman representation in quantum theory give direct meaning to an entire analytic function as the wave function of quantum states. Solutions of the planar electrons problem in magnetic field (Landau levels) and the Quantum Hall effect (the Laughlin wave function) include an arbitrary analytic wave function, which reflects degeneracy of the ground state.

In attempts to construct axiomatic quantum field theory analyticity plays the central role. The causality as one of the most fundamental principle of modern physics becomes related to analyticity principle and is reflected in the dispersion relations. Introduced in optics by Kronig and Kramers in 1926, these relations were intensively investigated in 1960s, when the concept of the $S$-matrix dominated in particle physics. The possibility to solve problem exactly just from symmetry principles has been realized then in the conformal field theory, one of the most active area of recent research in mathematical physics. It has found applications in string theory, statistical physics, phase transitions, condensed matter physics etc. Conformal mappings as a powerful method of solving boundary value problems in hydrodynamics and electrostatics, become an infinite dimensional group of quantum transformations.

In quantum scattering theory, analyticity plays a fundamental role and allows the formulation of integral equations for inverse scattering theory, like Gelfand-Levitan and Marchenko equations. Beautiful applications of this theory for solution of nonlinear evolution equations like the Korteweg-deVries equation, opened new area of applied mathematics as the soliton theory. Then an attempt to solve equations in $2+1$ dimension exactly, when quite difficult and some time impossible to specify analyticity domain for scattering data, leaded to extension of class of analytic functions to the generalized analytic functions. It was formulated as the $\bar{\partial}$-problem. This way generalized analytic functions become important for solution of higher dimensional evolution equations and some boundary value problems. Exact solutions of nonlinear field equations like solitons, instantons, cnoidal waves etc are based on analytic functions theory.

Finally we like to emphasize the deep role of analyticity in science in general, where the possibility of extrapolation is related with possibility of prediction. As mentioned by Rene Thom: 'I believe it is still true, even now, strict quantitative prediction in science is associated with analytic continuation'. Get out of the field of analyticity and analytic continuation is not possible and there is no strict way of extrapolation and quantitative prediction [6].

\section{2. q-periodic analytic functions}

Development of infinite dimensional group theory, conformal field theory and quantum integrable systems, has illuminated from a new direction one of the classical subjects known as 
$q$-calculus [2]. Besides quantum groups and anyon physics, this calculus was found recently in the applications in the old classical problem of hydrodynamics in circular multiple connected domain [3]. Here $q$-periodic analytic functions allowed us to formulate the two circle theorem for irrotational and incompressible flow in double connected domain bounded by two circles $[4,5]$. Let $f(z)$ is the complex potential of the flow in plane, then with addition of two concentric circular cylinders with cross sections $C_{1}:|z|=r_{1}$ and $C_{2}:|z|=r_{2}$, the flow between cylinders becomes

$$
F(z)=\sum_{n=-\infty}^{\infty} f\left(q^{n} z\right)+\sum_{n=-\infty}^{\infty} \bar{f}\left(q^{n} \frac{r_{1}^{2}}{z}\right) .
$$

Here parameter $q$ has the simple geometrical meaning $q=r_{2}^{2} / r_{1}^{2}$ as a unique characteristic of the double connected domain. This solution shows that complex potential is $q$-periodic analytic function $F(q z)=F(z)$. Corresponding complex velocity $\bar{V}(z)=\mathrm{d} F(z) / \mathrm{d} z$ is scaleinvariant analytic function $\bar{V}(q z)=q^{-1} \bar{V}(z)$ and admits representation $\bar{V}(z)=z^{-1} A_{q}(z)$, where $A_{q}(q z)=A_{q}(z)$ is $q$-periodic analytic function. The form of scale-invariant function $W(q t)=q^{d} A_{q}(t)$ is characteristic of fractal self-similar functions. So the famous MandelbrotWeierstrass fractal function is represented in this form. Besides in hydrodynamics, analytical extension of this function in Fock-Bargman or in coherent state representation can be used for construction of quantum wave function analogue of everywhere continuous but nowhere differentiable function of Mandelbrot-Weierstrass fractal. This is why naturally to consider it as quantum fractal. The structure of quantum fractal is typical for hierarchical lattices and phase transitions critical phenomena [20].

Due to the important role of analytic function theory in physics, any development in the concept of analytic functions could be highly desirable not only from the mathematical point of view but also for applications. Motivated by the hydrodynamic problem mentioned above with $q$-periodic and self-similar structure, in the present paper we study complex functions under finite scaling transformations. This allows us to introduce a new class of complex functions of complex argument, depending on real parameter $q$ and reducible to analytic functions for particular value of $q=1$. The construction is based on $q$-derivative extension of the Riemann holomorphicity equation. As an example of this $q$-analytic functions we treat in details the complex $q$-binomial. We show that this $q$-analytic function, being non-analytic for $q \neq 1$, still is the generalized analytic function. This function allows us to construct also new type of quantum coherent states and quantum fractals.

The paper is organized as follows. In section 2 we introduce main definitions and examples of $q$-analytic functions. $q$-Taylor expansion and $q$-Laurent expansions are considered in sections 3-5 correspondingly. The advantage of our approach is that due to factorization property of $q$-binomials, we have factorization of complex $q$-exponential functions, similar to the polar coordinate representation of a complex function. In section 6 we introduce $q$-harmonic functions. In section 7 we show that complex $q$-binomial is a generalized analytic function, satisfying D-Bar equation and the generalized Cauchy representation. Details of calculations are given in the appendix. In section 8 we consider self-similarity equation and construct complex $q$-analytic fractals represented in terms of double Mellin series. Sections 9 and 10 are devoted to application of $q$-analytic functions in quantum theory. In our presentation we try to be pedagogical and represent our ideas and calculations in explicit and detailed form.

\section{2. q-analytic function}

The $q$-differential of finite scale transformation for real function of one variable is defined as [2]

$$
\mathrm{d}_{q} f(x)=f(q x)-f(x)=\left(D_{x} f(x)\right) \mathrm{d}_{q} x,
$$


where $\mathrm{d}_{q} x=(q-1) x$, and $q$-derivative is

$$
D_{x} f(x)=\frac{f(q x)-f(x)}{(q-1) x} .
$$

For a complex-valued function $f(x, y)$ of two real variables $x$ and $y$, the $q$-differential

$$
\mathrm{d}_{q} f(x, y)=f(q x, q y)-f(x, y)
$$

can be rewritten as

$$
\mathrm{d}_{q} f(x, y)=\left(M_{q}^{y} D_{x} f(x, y)\right) \mathrm{d}_{q} x+\left(D_{y} f(x, y)\right) \mathrm{d}_{q} y,
$$

where $\mathrm{d}_{q} x=(q-1) x, \mathrm{~d}_{q} y=(q-1) y, D_{x}$ and $D_{y}$ are partial $q$-derivatives in $x$ and $y$ variables. Here $M_{q}^{y}$ is the dilatation operator in $y$ variable: $M_{q}^{y} F(x, y)=F(x, q y)$. In operator form we have $M_{q}^{y}=q^{y \frac{\mathrm{d}}{\mathrm{d} y}}$, and $D_{y} \equiv D_{q}^{y}=\frac{q^{y} \frac{\mathrm{d}}{\mathrm{d} y}-1}{(q-1) y}$. In terms of complex coordinates $z=x+\mathrm{i} y, \bar{z}=x-\mathrm{i} y$ we can rewrite complex $q$-differentials $\mathrm{d}_{q} z=\mathrm{d}_{q} x+\mathrm{id}_{q} y, \mathrm{~d}_{q} \bar{z}=\mathrm{d}_{q} x-\mathrm{id}_{q} y$ as $\mathrm{d}_{q} z=(q-1) \mathrm{d} z$ and $\mathrm{d}_{q} \bar{z}=(q-1) \mathrm{d} \bar{z}$. For $q$-differential of an arbitrary complex-valued function $f(x, y)$ then we get

$$
\mathrm{d}_{q} f(x, y)=\left(M_{q}^{y} D_{z} f\right) \mathrm{d}_{q} z+\left(M_{q}^{y} D_{\bar{z}} f\right) \mathrm{d}_{q} \bar{z}
$$

where we have introduced two linear operators of complex $q$-derivatives

$$
D_{z} \equiv \frac{1}{2}\left(D_{x}-\mathrm{i} M_{1 / q}^{y} D_{y}\right), \quad D_{\bar{z}} \equiv \frac{1}{2}\left(D_{x}+\mathrm{i} M_{1 / q}^{y} D_{y}\right) .
$$

Definition 2.1. A complex-valued function $f(x, y)$ of two real variables is called a $q$-analytic in a region if the following identity holds in the region:

$$
D_{\bar{z}} f=\frac{1}{2}\left(D_{x}+\mathrm{i} M_{1 / q}^{y} D_{y}\right) f=0 .
$$

The $q$-differential of $q$-analytic function then is given by

$$
\mathrm{d}_{q} f=\left(M_{q}^{y} D_{z} f\right) \mathrm{d}_{q} z
$$

In the limit $q \rightarrow 1$, this definition reduces to the standard analyticity condition

$$
\frac{\partial}{\partial \bar{z}} f=\frac{1}{2}\left(\frac{\partial}{\partial x}+\mathrm{i} \frac{\partial}{\partial y}\right) f=0
$$

leading to independence of $\bar{z}: d f=\frac{\partial f}{\partial z} \mathrm{~d} z$.

Similar way for $q$-anti-analytic function we have

$$
D_{z} f=\frac{1}{2}\left(D_{x}-\mathrm{i} M_{1 / q}^{y} D_{y}\right) f=0,
$$

and

$$
\mathrm{d}_{q} f=\left(M_{q}^{y} D_{\bar{z}} f\right) \mathrm{d}_{q} \bar{z} .
$$

We like to notice that analytic function $f(z)$ as a function of $z$ can depends on several constants. In case of $q$-holomorphic function (8) these constants could be arbitrary $q$-periodic functions of $z$. For example $D_{\bar{z}} f(z)=0$ determines $f(z)$ not uniquely but up to $f(z)+A_{q}(\bar{z})$, where $D_{\bar{z}} A_{q}(\bar{z})=0$, and $A_{q}(\bar{z})$-is $q$-periodic function $A_{q}(q \bar{z})=A_{q}(\bar{z})$. 


\section{1. q-analytic binomial}

The simplest and most important set of $q$-analytic functions is given by complex $q$-binomials $(x+\mathrm{i} y)_{q}^{n} \equiv(x+\mathrm{i} y)(x+\mathrm{i} q y)\left(x+\mathrm{i} q^{2} y\right) \ldots\left(x+\mathrm{i} q^{n-1} y\right)=\sum_{k=0}^{n}\left[\begin{array}{l}n \\ k\end{array}\right]_{q} q^{k(k-1) / 2} i^{k} x^{n-k} y^{k}$

expandable according to Gauss's binomial formula. Here we follow notations for real $q$-binomial introduced in [2]. By direct substitution we have

$$
D_{\bar{z}}(x+\mathrm{i} y)_{q}^{n}=0
$$

and

$$
D_{z}(x+\mathrm{i} y)_{q}^{n}=[n]_{q}(x+\mathrm{i} y)_{q}^{n-1} .
$$

Then for $q$-differential we get

$$
\mathrm{d}_{q}(x+\mathrm{i} y)_{q}^{n}=\left(M_{q}^{y} D_{z}(x+\mathrm{i} y)_{q}^{n}\right) \mathrm{d}_{q} z=[n]_{q}(x+\mathrm{i} q y)_{q}^{n-1} \mathrm{~d}_{q} z .
$$

Similar way it is easy to show that complex conjugate $q$-binomial $(x-\mathrm{i} y)_{q}^{n}$ is $q$-anti-analytic.

Here we notice an interesting limit of this binomial. For $q<1$ and $x=1$ the limit $n \rightarrow \infty$ exists and is given by the $q$-analogue of the Euler Formula

$$
(1+\mathrm{i} y)_{q}^{\infty}=E_{q}^{\mathrm{i} y /(1-q)}=\operatorname{Cos}_{q} \frac{y}{1-q}+\mathrm{i}^{\operatorname{Sin}_{q}} \frac{y}{1-q},
$$

where $E_{q}^{x}$ is the second Jackson's $q$-exponential function.

\subsection{Negative power q-analytic binomial}

We define complex $q$-binomial of negative power as

$$
(x+\mathrm{i} y)_{q}^{-n}=\frac{1}{\left(x+\mathrm{i} q^{-n} y\right)_{q}^{n}} .
$$

For $z \neq 0$ it is $q$-analytic function

$$
D_{\bar{z}}(x+\mathrm{i} y)_{q}^{-n}=0
$$

and

$$
D_{z}(x+\mathrm{i} y)_{q}^{-n}=[-n]_{q}(x+\mathrm{i} y)_{q}^{-(n+1)} .
$$

For $q$-differential then we have

$$
\mathrm{d}_{q}(x+\mathrm{i} y)_{q}^{-n}=\left(M_{q}^{y} D_{z}(x+\mathrm{i} y)_{q}^{-n}\right) \mathrm{d}_{q} z=[-n]_{q}(x+\mathrm{i} q y)_{q}^{-(n+1)} \mathrm{d}_{q} z .
$$

\section{3. $q$-Taylor formula for $q$-analytic polynomial}

By taking linear combination of complex $q$-binomials, we get $q$-analytic polynomials. Conversely, any complex-valued $q$-analytic polynomial function $P(z ; q)$ of degree $N$ has the following $q$-Taylor expansion

$$
P(z ; q)=\sum_{k=0}^{N}\left(D_{z}^{k} P\right)(0) \frac{(x+\mathrm{i} y)_{q}^{k}}{[k] !}
$$

It follows from expansion

$$
P(z ; q)=\sum_{k=0}^{N} a_{k}(x+\mathrm{i} y)_{q}^{k}
$$


where polynomials $\left\{(x+\mathrm{i} y),(x+\mathrm{i} y)_{q}^{2}, \ldots,(x+\mathrm{i} y)_{q}^{N}\right\}$ are linearly independent. They constitute a basis for the space of complex $q$-analytic polynomials degree $N$. Due to $q$-analyticity condition, the above expansion includes only $(x+\mathrm{iy})_{q}^{k}$ polynomials, and not the complex conjugate ones. Then differentiating this expression $k$-times in $z$, and putting $z=0$ we find coefficients $a_{k}=\left(D_{z}^{k} P\right)(0) /[k]$ !.

\section{4. $q$-Taylor representation for $q$-analytic functions}

In the limit $N \rightarrow \infty$, the above Taylor formula for convergent series, represents the $q$-analytic function

$$
f(z ; q)=\sum_{k=0}^{\infty} a_{k}(x+\mathrm{i} y)_{q}^{k}=\sum_{k=0}^{\infty}\left(D_{z}^{k} f\right)(0) \frac{(x+\mathrm{i} y)_{q}^{k}}{[k] !} .
$$

It is clear that this $q$-analytic function satisfies equation (8): $D_{\bar{z}} f(z)=0$. If we fix base $|q|<1$, and $n=0,1,2, \ldots$ then we get inequality

$$
x^{2}+q^{2 n} y^{2} \leqslant x^{2}+y^{2}
$$

which implies

$$
\left|(x+\mathrm{i} y)_{q}^{n}\right| \leqslant\left|(x+\mathrm{i} y)^{n}\right| \Rightarrow\left|\sum_{n=0}^{\infty} a_{n}(x+\mathrm{i} y)_{q}^{n}\right| \leqslant \sum_{n=0}^{\infty}\left|a_{n}\right|\left|(x+\mathrm{i} y)^{n}\right| .
$$

It gives next result.

Proposition 4.1. For a given complex-valued function $f(z)$ analytic inside the disc of radius $R, C_{R}:|z|<R, \partial f(z) / \partial \bar{z}=0$, and with the Taylor expansion

$$
f(z)=\sum_{n=0}^{\infty} a_{n} z^{n}
$$

exists the q-analytic function $f(z ; q), D_{\bar{z}} f(z ; q)=0$, convergent in the same disc $C_{R}$ with the $q$-Taylor expansion

$$
f(z ; q)=\sum_{n=0}^{\infty} a_{n}(x+\mathrm{i} y)_{q}^{n}
$$

According to this, to every analytic function corresponds the $q$-analytic function. For $q=1$ a $q$-analytic function becomes the analytic $f(z ; q=1)=f(z)$ and parameter $q$ shows deviation from this analyticity.

\section{1. q-analytic function examples}

From standard exponential and trigonometric functions we have following entire $q$-analytic functions with $|q|<1$ :

$$
\begin{aligned}
& e(z ; q)=\sum_{n=0}^{\infty} \frac{(x+\mathrm{i} y)_{q}^{n}}{n !} \\
& \sin (z ; q)=\sum_{n=0}^{\infty}(-1)^{n} \frac{(x+\mathrm{i} y)_{q}^{2 n+1}}{(2 n+1) !} \\
& \cos (z ; q)=\sum_{n=0}^{\infty}(-1)^{n} \frac{(x+\mathrm{i} y)_{q}^{2 n}}{(2 n) !}
\end{aligned}
$$


From the definition of Jackson's $q$-exponential function,

$$
e_{q}(z) \equiv \sum_{n=0}^{\infty} \frac{(x+\mathrm{i} y)^{n}}{[n] !_{q}}
$$

which is the entire function for $q>1$, we have $q$-analytic $q$-exponential function

$$
e_{q}(z ; q) \equiv \sum_{n=0}^{\infty} \frac{(x+\mathrm{i} y)_{q}^{n}}{[n] !_{q}}
$$

or in terms of $z \equiv x+\mathrm{i} y, \quad z_{q} \equiv x+\mathrm{i} q y, \quad \ldots \quad z_{q^{n}} \equiv x+\mathrm{i} q^{n} y, \ldots$,

$$
e_{q}(z ; q)=\sum_{n=0}^{\infty} \frac{z z_{q} \ldots z_{q^{n-1}}}{[n]_{q} !} .
$$

This function $e_{q}(x+\mathrm{i} y ; q)$ is $q$-analytic $D_{\bar{z}} e_{q}(z ; q)=0$ for $q>1$ in the strip $-\infty<x<\infty$, $|y|<q /(q-1)$, and can be factorized in terms of Jackson's $q$-exponential functions as

$$
e_{q}(x+\mathrm{i} y ; q)=e_{q}(x) E_{q}(\mathrm{i} y)=e_{q}(x)\left(\operatorname{Cos}_{q}(y)+\mathrm{i} \operatorname{Sin}_{q}(y)\right) .
$$

This formula is $q$-analogue of the Euler formula for analytic function $\mathrm{e}^{z}=\mathrm{e}^{x} \mathrm{e}^{\mathrm{i} y}=$ $\mathrm{e}^{x}(\cos y+\mathrm{i} \sin y)$.

Here we like to emphasize that $q$-analytic functions as complex-valued functions are not analytic functions in the usual sense, because arguments

$$
z_{q^{n}}=x+\mathrm{i} q^{n} y=\frac{\left(1+q^{n}\right)}{2} z+\frac{\left(1-q^{n}\right)}{2} \bar{z},
$$

include both $z$ and $\bar{z}$, so that $\frac{\partial}{\partial \bar{z}} e_{q}(x+\mathrm{i} y ; q) \neq 0$. The only exception for $q \neq 1$ is a linear function $f=a z+b$.

Geometrically, we can represent every complex variable $z_{q^{n}}=x+\mathrm{i} q^{n} y, n=$ $0, \pm 1, \pm 2, \ldots$ as a complex plane with coordinates $\left(x, q^{n} y\right)$ (with re-scaled $y$ coordinate). All these planes are intersecting along the real axis $x$. Then, the $q$-analytic function depends on infinite set of complex variables on these planes $z, z_{q^{ \pm 1}}, z_{q^{ \pm 2}}, \ldots$ and not on $\bar{z}, \bar{z}_{q^{ \pm 1}}, \bar{z}_{q^{ \pm 2}}, \ldots$ In the limiting case $q \rightarrow 1$, all planes coincide with the complex plane $z$, and $q$-analytic function becomes the standard analytic function.

\section{5. $q$-Laurent expansion for $q$-analytic functions}

The Laurent formula for an analytic function in annular domain allows us to introduce corresponding $q$-analytic function.

In (16) for the negative power $q$-binomial

$$
(x+\mathrm{i} y)_{q}^{-n}=\frac{1}{\left(x+\mathrm{i} q^{-n} y\right)_{q}^{n}},
$$

we found that for $z \neq 0$ it is $q$-analytic function, $D_{\bar{z}}(x+\mathrm{i} y)_{q}^{-n}=0$. If we fix the base $|q|<1$, then we have inequality

$$
\frac{1}{\left|\left(x+\mathrm{i} q^{-n} y\right)_{q}^{n}\right|} \leqslant \frac{1}{\left|(x+\mathrm{i} y)^{n}\right|}
$$

and as follows

$$
\left|\sum_{n=1}^{\infty} \frac{b_{n}}{\left(x+\mathrm{i} q^{-n} y\right)_{q}^{n}}\right| \leqslant \sum_{n=1}^{\infty} \frac{\left|b_{n}\right|}{\left|(x+\mathrm{i} y)^{n}\right|} .
$$

According to this relation we can extend class of $q$-analytic functions. 
Proposition 5.1. For a given complex-valued function $f(z)$ analytic inside the annular domain $D: r<|z|<R, \partial f(z) / \partial \bar{z}=0$, and with the Laurent expansion

$$
f(z)=\sum_{n=-\infty}^{\infty} b_{n} z^{n}
$$

exists the $q$-analytic function $f(z ; q), D_{\bar{z}} f(z ; q)=0$, convergent in the same domain $D$, with the q-Laurent expansion

$$
f(z ; q)=\sum_{n=-\infty}^{\infty} b_{n}(x+\mathrm{i} y)_{q}^{n}
$$

As an example we have

$$
e\left(\frac{1}{z} ; q\right)=\sum_{n=0}^{\infty} \frac{(x+\mathrm{i} y)_{q}^{-n}}{n !}=\sum_{n=0}^{\infty} \frac{1}{n !\left(x+\mathrm{i} q^{-n} y\right)_{q}^{n}}
$$

which is $q$-analytic everywhere except $z=0$.

\section{The $q$-Cauchy-Riemann equations}

Expanding a $q$-holomorphic function to real and imaginary parts $f(x+\mathrm{i} y ; q)=u(x, y ; q)+$ $\mathrm{i} v(x, y ; q)$ due to $(8),\left(D_{x}+\mathrm{i} M_{\frac{1}{q}}^{y} D_{y}\right)(u+\mathrm{i} v)=0$ we get the system of $q$-Cauchy-Riemann Equations

$$
D_{x} u=M_{\frac{1}{q}}^{y} D_{y} v, \quad D_{x} v=-M_{\frac{1}{q}}^{y} D_{y} u .
$$

The $q$-Laplace operator is defined in terms of $q$-holomorphic derivatives (7) as

$$
\Delta_{q} \equiv 4 D_{z} D_{\bar{z}}=D_{x}^{2}+\left(M_{\frac{1}{q}}^{y} D_{y}\right)^{2}=D_{x}^{2}+\frac{1}{q} M_{\frac{1}{q^{2}}}^{y} D_{y}^{2},
$$

where the order of $M_{q}^{y}$ and $D_{y}$ operators is interchanged according to $Q$-commutative formula $\left(D_{y} M_{Q}^{y}\right)=Q\left(M_{Q}^{y} D_{y}\right)$.

Due to (8) operator $D_{z}$ acts on $q$-holomorphic function $f(z ; q)$ just as $D_{x}$ derivative:

$$
D_{z} f(z ; q)=\frac{1}{2}\left(D_{x}-\mathrm{i} M_{\frac{1}{q}}^{y} D_{y}\right) f(z ; q)=D_{x} f(z ; q) .
$$

Definition 6.1. The real function $\phi(x, y)$ is a q-harmonic function if it satisfies the q-Laplace equation

$$
\Delta_{q} \phi(x, y)=0
$$

Due to factorization $\Delta_{q} f=4 D_{z} D_{\bar{z}} f=0$, the real and imaginary parts of a $q$-analytic function are conjugate $q$-harmonic functions

$$
\Delta_{q} u(x, y)=0, \quad \Delta_{q} v(x, y)=0 .
$$

These functions can be used for solution of two-dimensional $q$-heat and $q$-Schrödinger equations. Recently, we have studied the $q$-heat equations in a line [12,13]. Different forms of these equations can be derived in problems of random walk on quantum group [25] and gauge theory of self-similar systems [23]. Two-dimensional version of stationary heat distribution in such systems is described by the $q$-Laplace equation $\Delta_{q} u=0$ with general solution in terms of $q$-harmonic functions. 


\subsection{Examples of q-harmonic functions}

From $q$-binomial for $n=2$

$$
(x+\mathrm{i} y)_{q}^{2}=(x+\mathrm{i} y)(x+\mathrm{i} q y)=x^{2}-q y^{2}+(1+q) \mathrm{i} x y
$$

we have $q$-harmonically conjugate functions $u(x, y)=x^{2}-q y^{2}, v(x, y)=(1+q) x y$.

For arbitrary $n=1,2,3, \ldots$, polynomial $q$-harmonic functions are

$u(x, y)=\frac{1}{2}\left[(x+\mathrm{i} y)_{q}^{n}+(x-\mathrm{i} y)_{q}^{n}\right], \quad v(x, y)=\frac{1}{2 \mathrm{i}}\left[(x+\mathrm{i} y)_{q}^{n}-(x-\mathrm{i} y)_{q}^{n}\right]$.

Simplest non-polynomial $q$-harmonic functions follow from (28) as

$$
u(x, y)=e_{q}(x) \operatorname{Cos}_{q}(y), \quad v(x, y)=e_{q}(x) \operatorname{Sin}_{q}(y) .
$$

\section{7. q-analytic function as generalized analytic function}

In previous sections we have seen that $q$-analytic functions depend on both $z$ and $\bar{z}$ variables and are not analytic. Nevertheless, here we are going to show that some of $q$-analytic functions are generalized analytic functions [14]. This class of functions is related with the $\bar{\partial}$-problem (D-Bar problem). The scalar equation

$$
\frac{\partial \Phi(z, \bar{z})}{\partial \bar{z}}=f(z, \bar{z})
$$

for simple connected domain in complex z-plane called $\bar{\partial}$-problem [16]. For complex functions

$$
\Phi=u+\mathrm{i} v, \quad f=\frac{g+\mathrm{i} h}{2}, \quad z=x+\mathrm{i} y
$$

it is equivalent to the system of a generalized Cauchy-Riemann equations

$$
\frac{\partial u}{\partial x}-\frac{\partial v}{\partial y}=g(x, y), \quad \frac{\partial u}{\partial y}+\frac{\partial v}{\partial x}=h(x, y) .
$$

In case of analytic functions, $g(x, y)=h(x, y)=0 \rightarrow f(x, y)=0$ it recovers the CauchyRiemann equations.

Definition 7.1. Complex function $\Phi(z, \bar{z})$ in a region $R$, satisfying equation

$$
\frac{\partial \Phi}{\partial \bar{z}}=A(z, \bar{z}) \Phi+B(z, \bar{z}) \bar{\Phi}
$$

is called the generalized analytic function.

In particular case $B=0$ it reduces to D-Bar equation

$$
\frac{\partial \Phi}{\partial \bar{z}}=A(z, \bar{z}) \Phi
$$

which can be solved in closed form $[14,16]$ as:

$$
\Phi(z, \bar{z})=\omega(z) \mathrm{e}^{\frac{1}{2 \pi \mathrm{i}} \iint_{D} \frac{A(\zeta, \bar{\zeta})}{\zeta-z} \mathrm{~d} \zeta \wedge \mathrm{d} \bar{\zeta}},
$$

where $\omega(z)$ is an arbitrary analytic function. First time this solution was obtained by Theodoresco in 1931 [15]. 


\subsection{Complex q-binomial}

Here we show that complex $q$-binomials $\Phi(z, \bar{z})=(x+\mathrm{i} y)_{q}^{n}$ are generalized analytic functions. Calculating partial derivatives

$$
\frac{\frac{\partial}{\partial x}(x+\mathrm{i} y)_{q}^{n}}{(x+\mathrm{i} y)_{q}^{n}}=\frac{\partial}{\partial x} \sum_{k=0}^{n-1} \ln \left(x+\mathrm{i} q^{k} y\right)=\sum_{k=0}^{n-1} \frac{1}{x+\mathrm{i} q^{k} y}
$$

we get

$$
\begin{aligned}
\frac{\partial}{\partial x}(x+\mathrm{i} y)_{q}^{n} & =(x+\mathrm{i} y)_{q}^{n} \sum_{k=0}^{n-1} \frac{1}{x+\mathrm{i} q^{k} y} \\
\frac{\partial}{\partial y}(x+\mathrm{i} y)_{q}^{n} & =(x+\mathrm{i} y)_{q}^{n} \sum_{k=0}^{n-1} \frac{i q^{k}}{x+\mathrm{i} q^{k} y}
\end{aligned}
$$

and

$$
\frac{\partial}{\partial \bar{z}}(x+\mathrm{i} y)_{q}^{n}=\frac{1-q}{2}(x+\mathrm{i} y)_{q}^{n} \sum_{k=0}^{n-1} \frac{[k]}{x+\mathrm{i} q^{k} y} .
$$

Therefore $\Phi(z, \bar{z})=(x+\mathrm{i} y)_{q}^{n}$ is the generalized analytic function satisfying $\bar{\partial}$-equation (43)

$$
\frac{\partial}{\partial \bar{z}} \Phi(z, \bar{z})=\Phi(z, \bar{z})(1-q) \sum_{k=0}^{n-1} \frac{[k]}{\left(1+q^{k}\right) z+\left(1-q^{k}\right) \bar{z}},
$$

where

$$
A(z, \bar{z})=(1-q) \sum_{k=1}^{n-1} \frac{[k]_{q}}{\left(1+q^{k}\right) z+\left(1-q^{k}\right) \bar{z}} .
$$

Here parameter $q$ expresses deviation from analyticity, and for $q=1 \rightarrow A(z, \bar{z}) \equiv 0$ and D-Bar equation ( $\bar{\partial}$-problem) reduces to the holomorphicity condition $\frac{\partial}{\partial \bar{z}} z^{n}=0$. By using (43) and (44) we find new representation for $q$-Binomial:

$(x+\mathrm{i} y)_{q}^{n}=\omega(z) \exp \left[\frac{1}{2 \pi \mathrm{i}} \iint_{D} \frac{1-q}{\zeta-z} \sum_{k=1}^{n-1} \frac{[k]_{q}}{\left(1+q^{k}\right) \zeta+\left(1-q^{k}\right) \bar{\zeta}} \mathrm{d} \zeta \wedge \mathrm{d} \bar{\zeta}\right]$,

where

$$
\omega(z)=\left(\frac{z}{2}\right)^{n} \prod_{k=0}^{n-1}\left(1+q^{k}\right)
$$

Details of these calculations are given in appendix. This representation shows explicitly relation between complex $q$-binomial $(x+\mathrm{i} y)_{q}^{n}$ and complex binomial $(x+\mathrm{i} y)^{n}=z^{n}$.

\section{Complex $q$-analytic fractals}

In this section we are going to construct self-similar fractal surface as a $q$-analytic function. In papers $[20,21]$ it was shown how generators of fractal and multi-fractal sets with discrete dilatation symmetries can be related to $q$-derivative operator. It was applied then to free energy of spin systems on hierarchical lattices [20,21] and irreversible dynamics on such lattices [22]. Key point is that singular part of critical spin systems on hierarchical lattices possesses discrete dilatation symmetry and satisfies the homogeneity relation. Following similar arguments here we consider complex $q$-derivative and $q$-analytic functions to obtain new type of fractal sets. 
We introduce complex-valued function $f(x, y)$, as homogeneous function degree $d$ :

$$
f(q x, q y)=q^{d} f(x, y) \text {. }
$$

The $q$-differential of this function is

$$
\mathrm{d}_{q} f=f(q x, q y)-f(x, y)=\left(q^{d}-1\right) f(x, y)
$$

and due to (6) it can be rewritten as

$$
\left(q^{d}-1\right) f(x, y)=\left(M_{q}^{y} D_{z} f\right) \mathrm{d}_{q} z+\left(M_{q}^{y} D_{\bar{z}} f\right) \mathrm{d}_{q} \bar{z} .
$$

For $q$-analytic function $D_{\bar{z}} f=0$ the last term vanishes and we have the homogeneous $q$-difference equation

$$
{ }_{z} M_{q}^{y} D_{z} f=\frac{q^{d}-1}{q-1} f .
$$

Below we consider only the case $d=n$ as a positive integer. To find solution of this equation, first we notice that complex $q$-binomial $(x+\mathrm{i} y)_{q}^{n}$ is a homogenous function degree $n$

$$
(\lambda x+\mathrm{i} \lambda y)_{q}^{n}=\lambda^{n}(x+\mathrm{i} y)_{q}^{n} .
$$

Combining this condition for $\lambda=q$ with $q$-analyticity condition $D_{\bar{z}}(x+\mathrm{i} y)_{q}^{n}=0$, we find that it satisfies equation (54)

$$
z M_{q}^{y} D_{z}(x+\mathrm{i} y)_{q}^{n}=[n]_{q}(x+\mathrm{i} y)_{q}^{n} .
$$

Then the general $q$-analytic fractal solution is

$$
f(x, y)=(x+\mathrm{i} y)_{q}^{n} A_{q}(x, y),
$$

where $A_{q}(q x, q y)=A_{q}(q x, y)=A_{q}(x, q y)=A_{q}(x, y)$ is complex-valued $q$-periodic function in both $x$ and $y$.

By choosing $A_{q}(x, y)$ as a real $q$-periodic function, we get the $q$-harmonic fractals as

$$
U(x, y)=u(x, y) A_{q}(x, y), \quad V(x, y)=v(x, y) A_{q}(x, y),
$$

where $u$ and $v$ are $q$-harmonic functions (38). Specific form of these fractals depends on structure of $A_{q}(x, y)$. To fix it we have the following Proposition.

Proposition 8.1. A q-periodic function can be represented in the general form

$$
A_{q}(x, y)=(x y)^{-s} \sum_{k=-\infty}^{\infty} \sum_{l=-\infty}^{\infty} q^{-s(k+l)} G\left(q^{k} x, q^{l} y\right)
$$

\section{Proof.}

$$
A_{q}(q x, y)=(q x y)^{-s} \sum_{k=-\infty}^{\infty} \sum_{l=-\infty}^{\infty} q^{-s(k+l)} G\left(q^{k+1} x, q^{l} y\right) .
$$

By replacing $k \mapsto k-1$, it is obvious that $A_{q}(q x, y)=A_{q}(x, y)$. Similarly, it is easy to see that $A_{q}(x, y)$ is $q$-periodic in $y$ argument as well.

According to the above proposition, the general $q$-analytic fractal solution of $q$-difference self-similarity equation (56) is

$$
f(x, y)=(x y)^{-s}(x+\mathrm{i} y)_{q}^{n} \sum_{k=-\infty}^{\infty} \sum_{l=-\infty}^{\infty} q^{-s(k+l)} G\left(q^{k} x, q^{l} y\right) .
$$




\subsection{Examples of q-periodic functions}

For $G(x, y)=\sin x \sin y$, from (59) we find

$$
A_{q}(x, y)=(x y)^{-s} \sum_{k=-\infty}^{\infty} \sum_{l=-\infty}^{\infty} \frac{\sin \left(q^{k} x\right) \sin \left(q^{l} y\right)}{q^{s(k+l)}} .
$$

With another choice $G(x, y)=\left(1-\mathrm{e}^{\mathrm{i} x}\right)\left(1-\mathrm{e}^{\mathrm{i} y}\right)$, we get

$$
A_{q}(x, y)=(x y)^{-s} \sum_{k=-\infty}^{\infty} \sum_{l=-\infty}^{\infty} \frac{\left(1-\mathrm{e}^{\mathrm{i} q^{k} x}\right)\left(1-\mathrm{e}^{\mathrm{i} q^{l} y}\right)}{q^{s(k+l)}} .
$$

This function can be separated to one-dimensional $q$-periodic functions $A(x)$ and $B(y)$,

$$
A_{q}(x, y)=x^{-s} \sum_{k=-\infty}^{\infty} \frac{1-\mathrm{e}^{\mathrm{i} q^{k} x}}{q^{s k}} y^{-s} \sum_{l=-\infty}^{\infty} \frac{1-\mathrm{e}^{\mathrm{i} q^{l} y}}{q^{s l}}=A(x) B(y),
$$

representing the $q$-periodic parts of the Weierstrass-Mandelbrot function. And the last function is a canonical example of the fractal curve.

\subsection{Double Mellin series expansion}

Below we restrict our consideration by $A_{q}(x, y)=A_{q}(x) B_{q}(y)$, where $A_{q}(q x)=$ $A_{q}(x), B_{q}(q x)=B_{q}(x)$ are $q$-periodic functions. Without loss of generality we consider $A_{q}(x)$ case only in details. By changing argument $\ln x=t$ and $\ln q=T$ we have

$$
A_{q}(q x)=A_{q}(x) \Rightarrow A_{q}\left(e^{T} e^{t}\right)=A_{q}\left(e^{t}\right)
$$

Denoting $A_{q}\left(e^{t}\right) \equiv F(t)$ we find that it is T-periodic, $F(t+T)=F(T)$, and can be expanded to Fourier series

$$
F(t)=\sum_{n=-\infty}^{\infty} c_{n} \mathrm{e}^{\mathrm{i} 2 \pi n t}
$$

with Fourier coefficients

$$
c_{n}=\frac{1}{T} \int_{0}^{T} F(t) \mathrm{e}^{\frac{-\mathrm{i} 2 \pi n t}{T}} \mathrm{~d} t .
$$

According to this, $q$-periodic function $A_{q}(x)$ can be represented by complex series (the Mellin series)

$$
A_{q}(x)=F(\ln x)=\sum_{n=-\infty}^{\infty} c_{n} x^{\frac{\mathrm{i} 2 \pi n}{\ln q}}
$$

where

$$
c_{n}=\frac{1}{\ln q} \int_{1}^{q} A_{q}(x) x^{\frac{-\mathrm{i} 2 \pi n}{\ln q}} \frac{\mathrm{d} x}{x} .
$$

In a similar way for $B_{q}(y)$ we have

$$
B_{q}(y)=\sum_{n=-\infty}^{\infty} \mathrm{d}_{n} y^{\frac{\mathrm{i} 2 \pi n}{\ln q}}
$$

with coefficients

$$
\mathrm{d}_{n}=\frac{1}{\ln q} \int_{1}^{q} B_{q}(y) y^{\frac{-\mathrm{i} 2 \pi n}{\ln q}} \frac{\mathrm{d} y}{y} .
$$


Combining together we get the double Mellin series representation of $q$-periodic function in the following form

$$
A_{q}(x, y)=\sum_{n=-\infty}^{\infty} \sum_{m=-\infty}^{\infty} c_{n} \mathrm{~d}_{m} x^{\frac{\mathrm{i} 2 \pi n}{\ln q}} y^{\frac{\mathrm{i} 2 \pi n}{\ln q}}
$$

By substituting to (57) and expanding $q$-binomial according to Gauss's Binomial formula, we obtain expansion of self-similar $q$-analytic function ( $q$-analytic fractal) to double Mellin series

$$
f(x, y)=\sum_{k=-\infty}^{\infty} \sum_{m=-\infty}^{\infty} c_{k} \mathrm{~d}_{m} \sum_{l=0}^{n}\left[\begin{array}{l}
n \\
l
\end{array}\right]_{q}^{l} q^{\frac{l(l-1)}{2}} x^{n-l+\frac{\mathrm{i} 2 \pi k}{\ln q}} y^{l+\frac{\mathrm{i} 2 \pi m}{\ln q}} .
$$

In case of fractal (61) the expansion is

$$
\begin{aligned}
f(x, y) & =A_{q}(x, y)(x+\mathrm{i} y)_{q}^{n} \\
& =\sum_{k=-\infty}^{\infty} \sum_{m=-\infty}^{\infty} \sum_{l=0}^{n}\left[\begin{array}{l}
n \\
l
\end{array}\right]_{q} i^{l} q^{\frac{l(l-1)}{2}} x^{n-l-s} y^{l-s} \frac{\sin \left(q^{k} x\right) \sin \left(q^{m} y\right)}{q^{s(k+m)}} .
\end{aligned}
$$

8.2.1. Examples of $q$-analytic fractals. By choosing function

$$
A_{q}(x, y)=\sin \left(\frac{2 \pi}{\ln q} \ln |x|\right) \cdot \sin \left(\frac{2 \pi}{\ln q} \ln |y|\right),
$$

as $q$-periodic in $x$ and $y$, we obtain the following set of homogenous self-similar $q$-analytic fractals of degree $n$,

$$
f_{n}(x, y)=\sin \left(\frac{2 \pi}{\ln q} \ln |x|\right) \cdot \sin \left(\frac{2 \pi}{\ln q} \ln |y|\right) \cdot(x+\mathrm{i} y)_{q}^{n},
$$

and for $\operatorname{Re} f_{n}(x, y) \equiv u_{n}$ and $\operatorname{Im} f_{n}(x, y) \equiv v_{n}$ the set of self-similar $q$-harmonic functions.

For $n=0$, the simplest $q$-harmonic and $q$-periodic function is $f_{0}(x, y)=A_{q}(x, y)$ from (66). In figures 1 and 2 , we plot $f_{0}(x, y)$ for $q=2$ and $-0.5 \leqslant x \leqslant 0.5,-0.5 \leqslant y \leqslant 0.5$. By changing scale $(x, y) \rightarrow\left(q^{n} x, q^{n} y\right)$, or in our example magnifying our figure in scales $\ldots, \frac{1}{4}, \frac{1}{2}, 2,4,8, \ldots$, etc we find that the figure shows the self-similar character remaining in the same form.

For $n=2$, we have

$$
\begin{aligned}
& u_{2}(x, y)=\left(x^{2}-q y^{2}\right) \cdot \sin \left(\frac{2 \pi}{\ln q} \ln |x|\right) \cdot \sin \left(\frac{2 \pi}{\ln q} \ln |y|\right), \\
& v_{2}(x, y)=[2]_{q}(x y) \cdot \sin \left(\frac{2 \pi}{\ln q} \ln |x|\right) \cdot \sin \left(\frac{2 \pi}{\ln q} \ln |y|\right) .
\end{aligned}
$$

In figure 3 we show $3 \mathrm{D}$ plot of $u_{2}(x, y)$ at $q=2$ and $-10 \leqslant x \leqslant 10,-10 \leqslant y \leqslant 10$. By re-scaling coordinates in $2^{n}$ scale we get the same figures, showing self-similar structure of our $q$-harmonic function.

For $n=3$, we get

$$
\begin{aligned}
& u(x, y)=x\left(x^{2}-q y^{2}-[2]_{q} q^{2} y^{2}\right) \cdot \sin \left(\frac{2 \pi}{\ln q} \ln |x|\right) \cdot \sin \left(\frac{2 \pi}{\ln q} \ln |y|\right), \\
& v(x, y)=y\left([2]_{q} x^{2}+q^{2}\left(x^{2}-q y^{2}\right)\right) \cdot \sin \left(\frac{2 \pi}{\ln q} \ln |x|\right) \cdot \sin \left(\frac{2 \pi}{\ln q} \ln |y|\right) .
\end{aligned}
$$

In figure 4 we show 3D plot of $q$-harmonic fractal $u_{3}(x, y)$ at $q=2$ and $-1 \leqslant x \leqslant 1$, $-1 \leqslant y \leqslant 1$. This figure also shows self-similar structure at $2^{n}$ scale. 


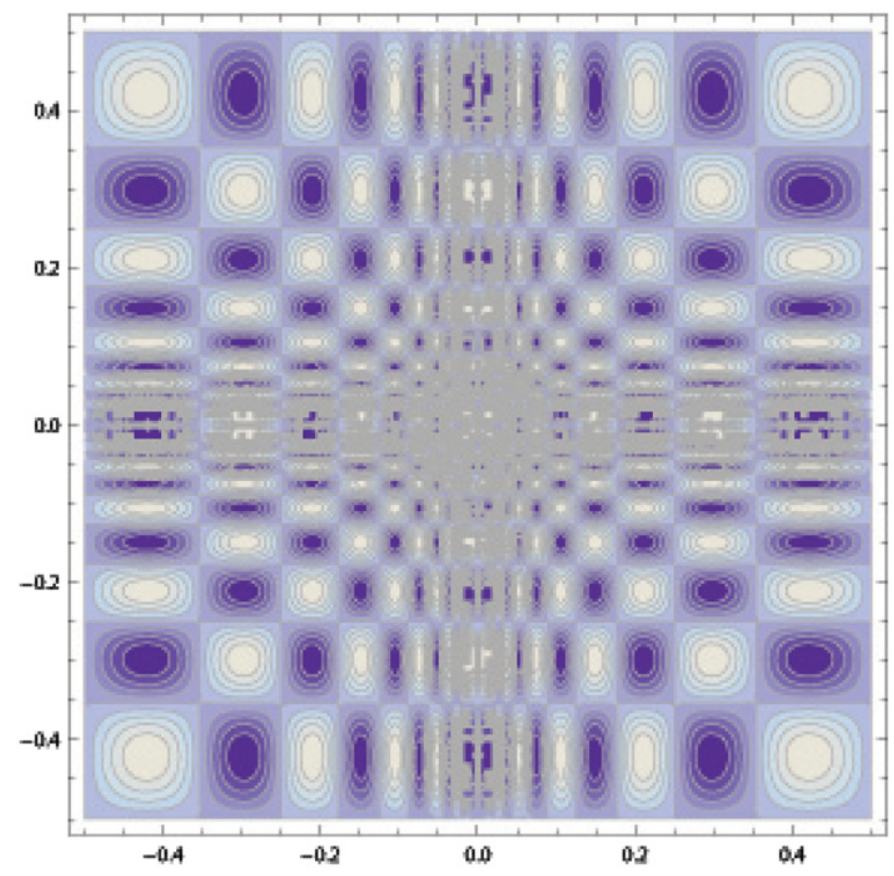

Figure 1. Contour plot of $q$-periodic $q$-harmonic function.

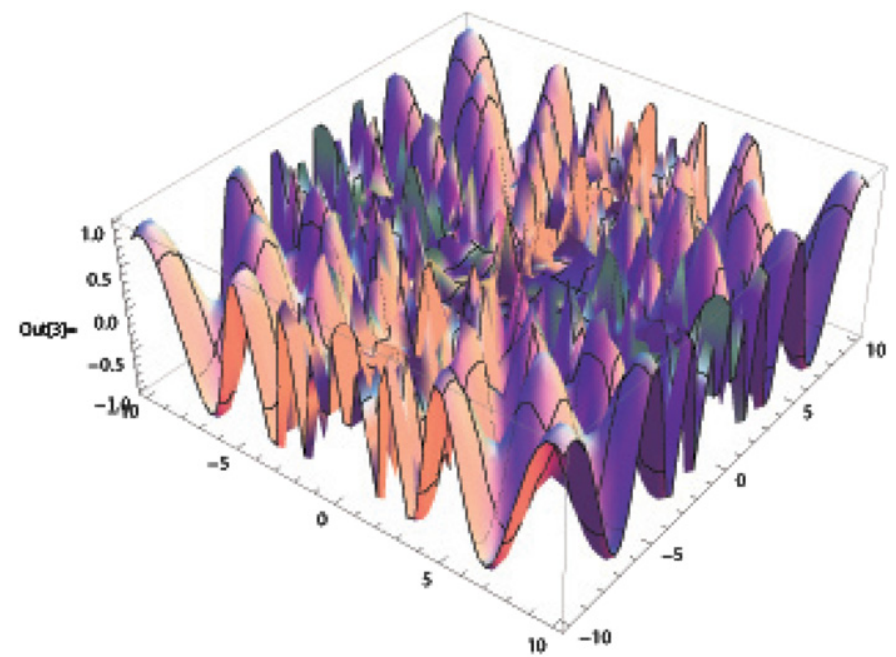

Figure 2. 3D plot of $q$-periodic $q$-harmonic function.

For $n=-1$, we have

$$
f_{-1}(x, y)=\sin \left(\frac{2 \pi}{\ln q} \ln |x|\right) \cdot \sin \left(\frac{2 \pi}{\ln q} \ln |y|\right) \cdot(x+\mathrm{i} y)_{q}^{-1}
$$




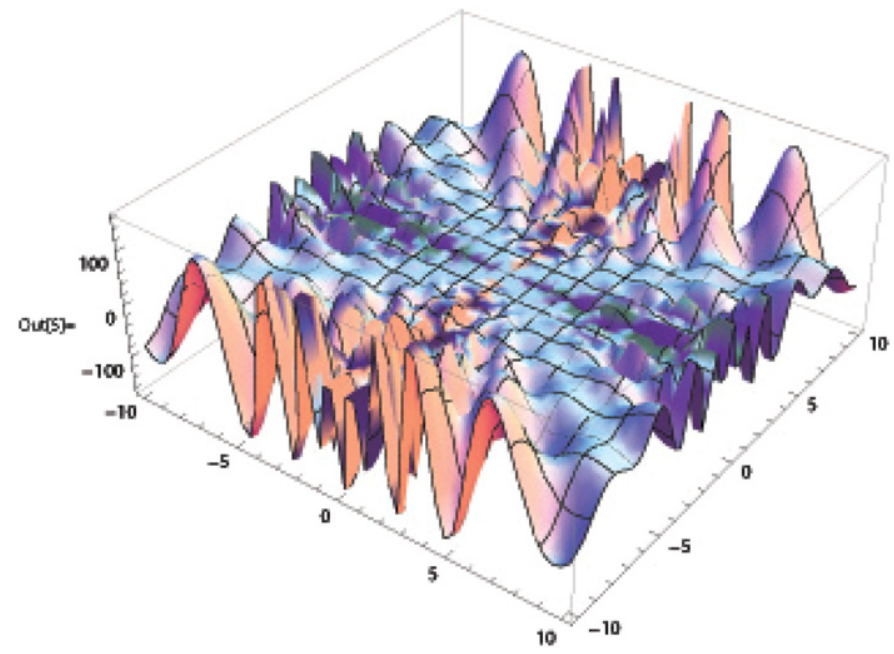

Figure 3. 3D plot of $n=2 q$-harmonic function.

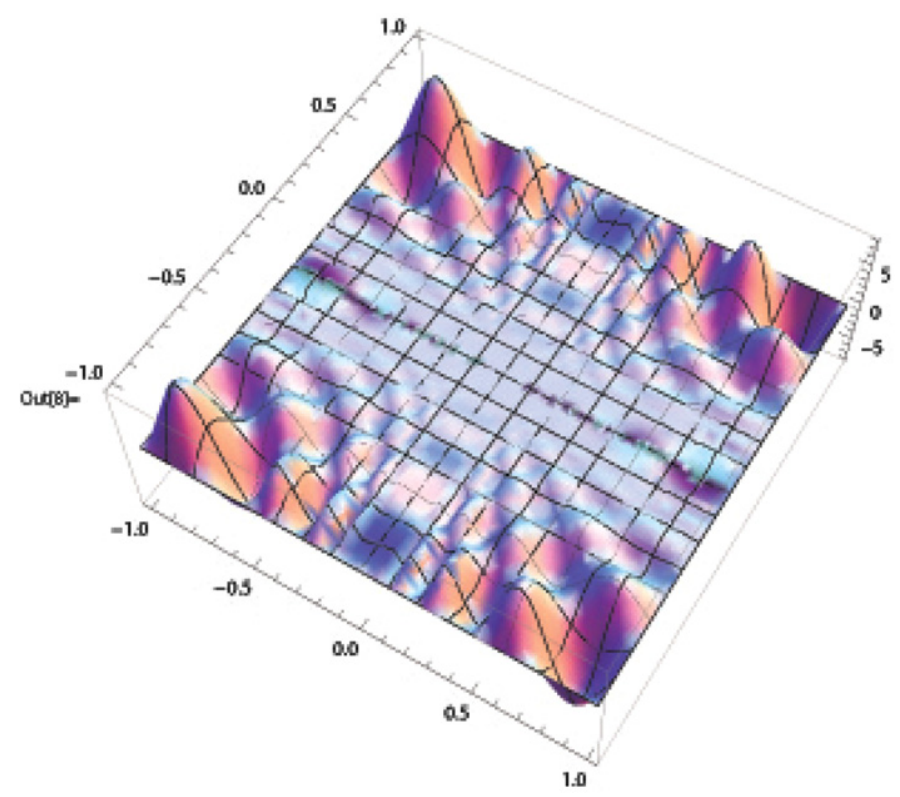

Figure 4. 3D plot of $n=3 q$-harmonic function.

and corresponding self-similar $q$-harmonic functions for $(x+\mathrm{i} y \neq 0)$ are

$$
\begin{aligned}
& u(x, y)=\frac{q^{2} x}{x^{2} q^{2}+y^{2}} \cdot \sin \left(\frac{2 \pi}{\ln q} \ln |x|\right) \cdot \sin \left(\frac{2 \pi}{\ln q} \ln |y|\right) \\
& v(x, y)=\frac{-q y}{x^{2} q^{2}+y^{2}} \cdot \sin \left(\frac{2 \pi}{\ln q} \ln |x|\right) \cdot \sin \left(\frac{2 \pi}{\ln q} \ln |y|\right)
\end{aligned}
$$

In figures 5 and 6 we show contour plot and 3D plot of this $q$-harmonic fractal $u(x, y)$ at $q=2$. 


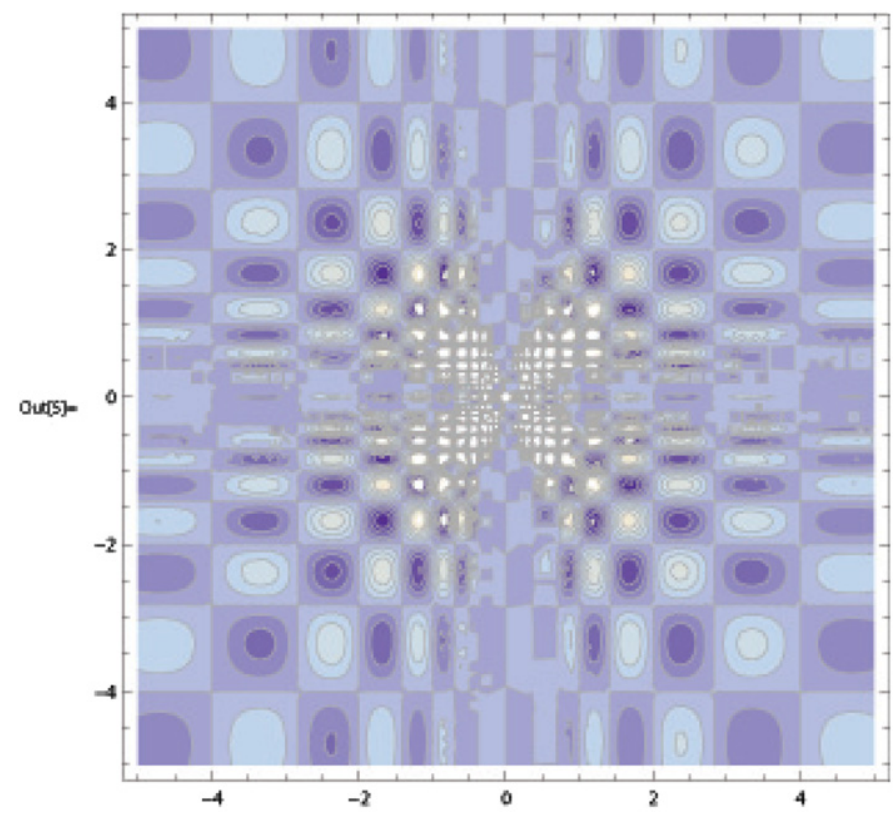

Figure 5. Contour plot of $n=-1 q$-harmonic function.

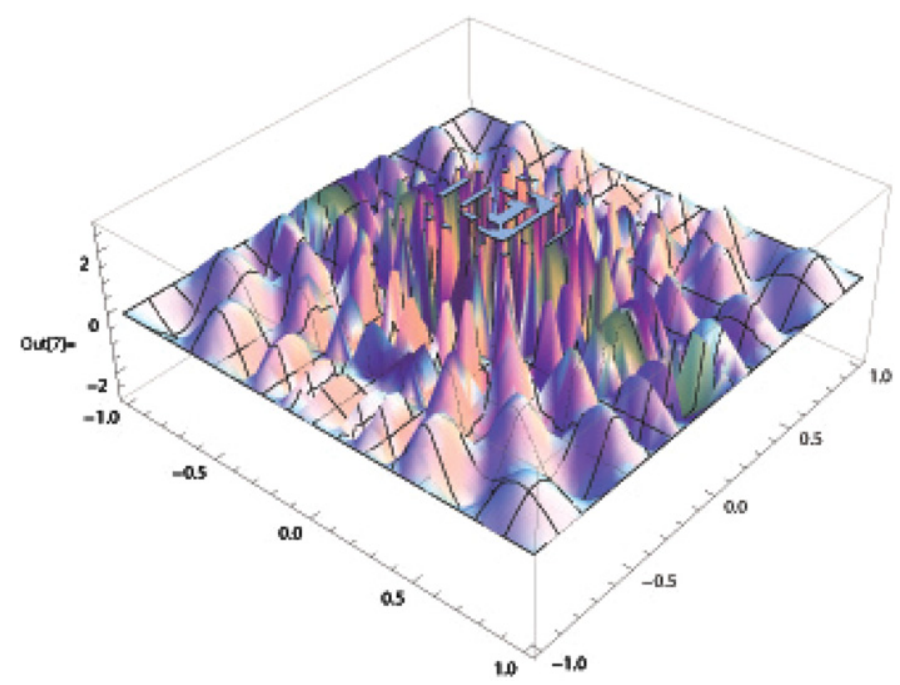

Figure 6. 3D plot of $n=-1 q$-harmonic function.

\section{9. q-analytic coherent states}

In this section, we apply our $q$-analytic functions to construct quantum states of harmonic oscillator. We consider bosonic operators

$$
\left[a, a^{+}\right]=I, \quad[a, I]=0, \quad\left[a^{+}, I\right]=0
$$


and the vacuum state $|0\rangle$ :

$$
a|0\rangle=0, \quad\langle 0 \mid 0\rangle=1 .
$$

The orthonormal set of $n$-particle states, $n=0,1,2, \ldots$,

$$
|n\rangle=\frac{\left(a^{+}\right)^{n}}{\sqrt{n !}}|0\rangle, \quad\langle n \mid m\rangle=\delta_{n m},
$$

generates the normalized Glauber coherent states, with complex $\alpha$ [27],

$$
|\alpha\rangle=\mathrm{e}^{-\frac{1}{2}|\alpha|^{2}} \sum_{n=0}^{\infty} \frac{\alpha^{n}}{\sqrt{n !}}|n\rangle
$$

By analogy with these coherent states we introduce a new set of $q$-analytic coherent states, parameterized by complex number $\alpha=\alpha_{1}+\mathrm{i} \alpha_{2}$ :

$$
|\alpha ; q\rangle=C \sum_{n=0}^{\infty} \frac{\left(\alpha_{1}+\mathrm{i} \alpha_{2}\right)_{q}^{n}}{\sqrt{[n]_{q} !}}|n\rangle .
$$

Normalization condition gives

$$
1=\langle\alpha ; q \mid \alpha ; q\rangle=|C|^{2} \sum_{n=0}^{\infty} \frac{\left(\alpha_{1}^{2}+\alpha_{2}^{2}\right)_{q^{2}}^{n}}{[n]_{q} !}=|C|^{2} e_{q}\left(\alpha_{1}^{2}+\alpha_{2}^{2}\right)_{q^{2}},
$$

where we denoted

$$
e_{q}\left(\alpha_{1}^{2}+\alpha_{2}^{2}\right)_{q^{2}}=\sum_{n=0}^{\infty} \frac{\left(\alpha_{1}^{2}+\alpha_{2}^{2}\right)_{q^{2}}^{n}}{[n]_{q} !}
$$

in Hahn's notations [8], see also [11]. Then the normalized $q$-analytic coherent states are given by

$$
|\alpha ; q\rangle=\left(e_{q}\left(\alpha_{1}^{2}+\alpha_{2}^{2}\right)_{q^{2}}\right)^{-\frac{1}{2}} \sum_{n=0}^{\infty} \frac{\left(\alpha_{1}+\mathrm{i} \alpha_{2}\right)_{q}^{n}}{\sqrt{[n]_{q} !}}|n\rangle .
$$

For $|q|<1$, due to evident relation $\left(\alpha_{1}^{2}+\alpha_{2}^{2}\right)_{q^{2}}^{n} \leqslant\left(\alpha_{1}^{2}+\alpha_{2}^{2}\right)^{n}$, we get inequality

$$
e_{q}\left(\alpha_{1}^{2}+\alpha_{2}^{2}\right)_{q^{2}} \leqslant e_{q}\left(\alpha_{1}^{2}+\alpha_{2}^{2}\right)
$$

where on the rhs we have the Jackson $q$-exponential function. From infinite product representation [2] of the last function

$$
e_{q}\left(|\alpha|^{2}\right)=\frac{1}{\left(1-(1-q)|\alpha|^{2}\right)_{q}^{\infty}}
$$

we can see that singularities of this function are located on the set of concentric circles with radiuses given by growing geometric progression $r_{n}=r_{0} q^{-n / 2}, r_{0}=1 / \sqrt{1-q}$. Then both functions convergent in the $\operatorname{disc} D:|\alpha|^{2} \leqslant 1 /(1-q)$. This is the region where normalization of our $q$-analytic coherent states is defined.

When $q \rightarrow 1$ these states reduce to the Glauber coherent states (75) and radius of convergency $r_{0} \rightarrow \infty$. Here we emphasize that our $q$-analytic coherent states are also different from the $q$-coherent states appearing in representation of $q$-deformed Heisenberg-Weyl algebra [17-19]. The last ones are analytic in $\alpha$, while our states are not analytic but the $q$-analytic. 


\section{0. $q$-analytic Fock-Bargmann representation}

The standard Fock-Bargman representation of an arbitrary state

$$
|\psi\rangle=\sum_{n=0}^{\infty} c_{n}|n\rangle, \quad\langle\psi \mid \psi\rangle=\sum_{n=0}^{\infty}\left|c_{n}\right|^{2}=1,
$$

is given by the scalar product of this state with Glauber's coherent state (75):

$$
\langle\alpha \mid \psi\rangle=\mathrm{e}^{-\frac{1}{2}|\alpha|^{2}} \psi(\bar{\alpha})
$$

where the wave function

$$
\psi(\alpha)=\sum_{n=0}^{\infty} c_{n} \frac{\alpha^{n}}{\sqrt{n !}}
$$

is an entire analytic function [27].

As an example, our $q$-analytic coherent state (78) in Fock-Bargman representation $\langle z \mid \alpha ; q\rangle$ is characterized by analytic function in $z$ :

$$
\psi_{\alpha}(z)=\left(e_{q}\left(\alpha_{1}^{2}+\alpha_{2}^{2}\right)_{q^{2}}\right)^{-\frac{1}{2}} \sum_{n=0}^{\infty} \frac{\left(\alpha_{1}+\mathrm{i} \alpha_{2}\right)_{q}^{n} z^{n}}{\sqrt{[n]_{q} ! n !}} .
$$

By using our $q$-analytic coherent states (78), now we introduce new representation of these states which we call the $q$-analytic Fock-Bargman representation. By taking the scalar product of $|\psi\rangle$ with (78) we get

$$
\begin{aligned}
\langle\alpha ; q \mid \psi\rangle & =\left(e_{q}\left(\alpha_{1}^{2}+\alpha_{2}^{2}\right)_{q^{2}}\right)^{-\frac{1}{2}} \sum_{n=0}^{\infty} c_{n} \frac{\left(\alpha_{1}-\mathrm{i} \alpha_{2}\right)_{q}^{n}}{\sqrt{[n]_{q} !}} \\
& =\left(e_{q}\left(\alpha_{1}^{2}+\alpha_{2}^{2}\right)_{q^{2}}\right)^{-\frac{1}{2}} \psi(\bar{\alpha} ; q)
\end{aligned}
$$

where the wave function

$$
\psi(\alpha ; q)=\sum_{n=0}^{\infty} c_{n} \frac{\left(\alpha_{1}+\mathrm{i} \alpha_{2}\right)_{q}^{n}}{\sqrt{[n]_{q} !}},
$$

is complex $q$-analytic function. Therefore, every complex $q$-analytic function, $D_{\bar{z}} \psi(x+\mathrm{i} y)_{q}=$ 0 , determines quantum state in our $q$-analytic Fock-Bargmann representation.

Proposition 4.1 from section 4, allows us to compare two wave functions in Fock-Bargman representation (82) and in $q$-analytic Fock-Bargman representation (86). Entire character of the first one implies existence of the second one for $|q|<1$.

As simplest example we find representation of the orthonormal basis $\{|n\rangle\}$, which is given just by complex $q$-analytic binomial

$$
|n\rangle \rightarrow \psi_{n}(\alpha ; q)=\frac{\left(\alpha_{1}+\mathrm{i} \alpha_{2}\right)_{q}^{n}}{\sqrt{[n]_{q} !}}
$$

It is not analytic, but as we have seen in section 6, it represents the generalized analytic function.

As a next example, we find the Glauber coherent state $|\alpha\rangle$ (75) in our $q$-analytic FockBargmann representation $\langle z ; q \mid \alpha\rangle$ :

$$
\psi_{\alpha}(z ; q)=\mathrm{e}^{-\frac{1}{2}|\alpha|^{2}} \sum_{n=0}^{\infty} \frac{(x+\mathrm{i} y)_{q}^{n}}{\sqrt{[n]_{q} !}} \frac{\alpha^{n}}{\sqrt{n !}}=\mathrm{e}^{-\frac{1}{2}|\alpha|^{2}} \sum_{n=0}^{\infty} \frac{(\alpha x+\mathrm{i} \alpha y)_{q}^{n}}{\sqrt{[n]_{q} ! n !}},
$$

which is $q$-analytic in $z=x+\mathrm{i} y$. 


\subsection{Quantum q-oscillator}

We consider $q$-bosons with creation and annihilation operators

$$
\begin{aligned}
& b^{+}=a^{+} \sqrt{\frac{[N+I]_{q}}{N+I}}=\sqrt{\frac{[N]_{q}}{N}} a^{+}, \\
& b=\sqrt{\frac{[N+I]_{q}}{N+I}} a=a \sqrt{\frac{[N]_{q}}{N}},
\end{aligned}
$$

where operators $a^{+}, a$ are given by (72), $N=a^{+} a,[N]_{q}=\frac{q^{N}-1}{q-1}$. The commutation relations are

$$
\begin{aligned}
& b b^{+}-b^{+} b=q^{N}, \\
& b b^{+}-q b^{+} b=I,
\end{aligned}
$$

and for $q$-number operators we have

$$
b^{+} b=[N]_{q}, \quad b b^{+}=[N+I]_{q} .
$$

As easy to see, $n$-particle states for $b$ and $a$ operators are the same

$$
|n\rangle=\frac{\left(a^{+}\right)^{n}}{\sqrt{n !}}|0\rangle=\frac{\left(b^{+}\right)^{n}}{\sqrt{[n]_{q} !}}|0\rangle,
$$

where vacuum state is $a|0\rangle=b|0\rangle=0$. Then for $b, b^{+}$operators we have

$$
b|n\rangle=\sqrt{[n]_{q}}|n-1\rangle, \quad b^{+}|n\rangle=\sqrt{[n+1]_{q}}|n+1\rangle .
$$

By using last relations we find action of these operators in $q$-analytic Fock-Bargman representation:

$$
b \rightarrow D_{z}, \quad b^{+} \rightarrow z M_{q}^{y}
$$

where $D_{z}$ is complex derivative operator defined in (7). For $q$-number operator we get representation

$$
[N]_{q} \rightarrow z M_{q}^{y} D_{z}
$$

This representation shows interesting connection with self-similarity condition discussed in section 6. The eigenvalue problem

$$
[N]_{q}|n\rangle=[n]_{q}|n\rangle
$$

in $q$-analytic Fock-Bargman representation

$$
z M_{q}^{y} D_{z} \frac{(x+\mathrm{i} y)_{q}^{n}}{\sqrt{[n]_{q} !}}=[n]_{q} \frac{(x+\mathrm{i} y)_{q}^{n}}{\sqrt{[n]_{q} !}},
$$

is equivalent to the self-similarity $q$-difference equation (56).

Quantum $q$-oscillator is described by Hamiltonian operator

$$
H=\hbar \omega\left(b b^{+}+b^{+} b\right) \text {. }
$$

The Hamiltonian in $q$-analytic FB representation becomes operator

$$
H=\hbar \omega\left(D_{z} z M_{q}^{y}+z M_{q}^{y} D_{z}\right),
$$

and the Schrödinger equation

$$
H|n\rangle=E_{n}|n\rangle,
$$


takes the form of $q$-difference equation

$$
\hbar \omega\left(D_{z} z M_{q}^{y}+z M_{q}^{y} D_{z}\right) \psi_{n}(z ; q)=E_{n} \psi_{n}(z ; q),
$$

with $q$-analytic solution

$$
\psi_{n}(z ; q)=\frac{(x+\mathrm{i} y)_{q}^{n}}{\sqrt{[n]_{q} !}}, \quad E_{n}=\hbar \omega\left([n]_{q}+[n+1]_{q}\right) .
$$

The above consideration shows that our $q$-analytic functions even being non-analytic functions could describe quantum states. Moreover, fractal $q$-analytic functions discussed in section 7 describe quantum states with fractal properties. These questions are now under investigation.

\section{Conclusion}

In the present paper we have introduced a new type of analyticity property for the complex function of the complex argument which we called $q$-analyticity. The main object in our consideration is given by the complex $q$-binomial as a $q$-analytic function. It was shown that this $q$-analytic function is not an analytic function in the standard sense, but is a generalized analytic function. We formulated a self-similar equation for the $q$-analytic function and constructed $q$-analytic fractals in terms of the $q$-periodic function and double Mellin series.

Following similar steps as in section 7 we can show that our $q$-analytic fractals are also generalized analytic functions. For example, the simplest $q$-periodic fractal (66) satisfies the D-Bar equation

$$
\frac{\partial}{\partial \bar{z}} \phi(z, \bar{z})=B(z, \bar{z}) \phi(z, \bar{z}),
$$

where $f_{0}(x, y) \equiv \phi(z, \bar{z})$ and

$$
\begin{aligned}
B(z, \bar{z})=(1-q) & \sum_{k=1}^{n-1} \frac{[k]_{q}}{\left(1+q^{k}\right) z+\left(1-q^{k}\right) \bar{z}} \\
& +\frac{4 \pi}{\ln q}\left(\frac{\cot \left(\frac{2 \pi}{\ln q} \ln \frac{|z+\bar{z}|}{2}\right)}{z+\bar{z}}-\frac{\cot \left(\frac{2 \pi}{\ln q} \ln \frac{|z-\bar{z}|}{2}\right)}{z-\bar{z}}\right) .
\end{aligned}
$$

Here we should comment an important point related with terminology and originality of our results comparing with other papers. First we should mention paper [9] which has introduced the $q$-complex numbers as 'a kind of complex numbers' based on abstract algebraic concept of $q$-addition. In contrast to it, in our paper we play with ordinary complex numbers and complex function theory. Moreover definition of complex $q$-difference operator and $q$ holomorphic equation in paper [9] and in our paper are different. Complex $q$-binomials which are main $q$-analytic example in our paper are not satisfying holomorphic condition in Ernst paper, so they are not holomorphic in his sense. There exists the set of polynomials satisfying $q$-holomorphicity condition in [9], but they do not have simple product representation as $q$ binomials. But this product representation is crucial for a function to be the generalized analytic function. We think that complex $q$-binomials are most natural complex extensions of $q$-Taylor expansion introduced by Jackson [7], which recently was applied to statistical field theory in [24] and to generalization of multifractal theory in [26]. This is why we prefer instead of abstract mathematical concepts to work on the solid basis of conventional complex analysis. The second paper [10], called as motivation for $q$-complex numbers, adds no new results comparing with the first paper, but contains basically the history of conventional calculus 
with piquant details. One more paper should be mentioned [28], where the notion of the $q$-analytic function is given to convergent double power series of $q$-commutative operators from the quantum plane. This definition has no direct relation with ours and do not use holomorphic equation at all, though we think it is possible to reformulate our results in terms of $q$-commutative coordinates. This questions would be addressed in our future work.

Finally we think that our results relating $q$-analytic functions with fractals and generalized analytic functions are new and important for several applications. The first potential field of applications is related with point vortex problems in bounded domains where $q$-calculus already showed usefulness by computing all necessary physical quantities like vortex images, Hamiltonian function, stream function, etc [3]. The second potential application is related to new type of $q$-analytic coherent states. This set of quantum states allows one to construct a new type of Fock-Bargmann representation with description of the $q$-oscillator in terms of $q$-analytic functions. We expect that by using this approach we can construct a new type of quantum fractal coherent states with self-similarity properties.

\section{Acknowledgments}

This work was carried out with support from TUBITAK (The Scientific and Technological Research Council of Turkey), TBAG Project $110 \mathrm{T679}$ and Izmir Institute of Technology. One of the authors (SN) was partially supported by TUBITAK scholarship for graduate students. The authors would like to thank referees for constructive comments.

\section{Appendix. D-Bar representation of complex q-binomial}

In this section, we are going to prove $q$-complex binomial representation (49), (50).

\section{A.1. Generalized Cauchy formula}

For non-analytic function $\Phi(z)$, the next generalized Cauchy formula is valid $[14,16]$

$$
\Phi(z)=\frac{1}{2 \pi \mathrm{i}} \oint_{\Gamma} \frac{\Phi(\zeta) \mathrm{d} \zeta}{\zeta-z}-\frac{1}{\pi} \iint_{G} \frac{\partial \Phi}{\partial \bar{\zeta}} \frac{\mathrm{d} \xi \mathrm{d} \eta}{\zeta-z}
$$

where $\zeta=\xi+\mathrm{i} \eta$. First we are going to check this formula for non-analytic function

with

$$
\Phi_{n}(z)=x+\mathrm{i} q^{n} y=\frac{1+q^{n}}{2} z+\frac{1-q^{n}}{2} \bar{z}
$$

$$
\frac{\partial \Phi_{n}}{\partial \bar{z}}=\frac{1-q^{n}}{2}=\frac{[n]_{q}}{2}(1-q)
$$

For the disc of radius $R$ we have:

(a) The line integral part in the above generalized Cauchy formula gives

$$
\begin{aligned}
\frac{1}{2 \pi \mathrm{i}} \oint_{\Gamma} \frac{\frac{1+q^{n}}{2} \zeta}{\zeta}+\frac{1-q^{n} \bar{\zeta}}{2} \mathrm{~d} \zeta=\frac{1}{2 \pi \mathrm{i}} \frac{1+q^{n}}{2} \oint_{\Gamma} \mathrm{d} \zeta \\
+\frac{1}{2 \pi \mathrm{i}} \frac{1+q^{n}}{2} z \oint_{\Gamma} \frac{\mathrm{d} \zeta}{\zeta-z}+\frac{1}{2 \pi \mathrm{i}} \frac{1-q^{n}}{2} \oint_{\Gamma} \frac{\bar{\zeta} \mathrm{d} \zeta}{\zeta-z} .
\end{aligned}
$$

The first integral vanishes, while the second one gives $2 \pi$ i so that we have

$$
\frac{1+q^{n}}{2} z+\frac{1}{2 \pi \mathrm{i}} \frac{1-q^{n}}{2} \oint_{\Gamma} \frac{\bar{\zeta} \mathrm{d} \zeta}{\zeta-z} .
$$


By substitution $\zeta=R \mathrm{e}^{\mathrm{i} \theta}$ the last integral becomes

$$
\frac{1}{2 \pi \mathrm{i}} \frac{1-q^{n}}{2} \int_{0}^{2 \pi} \frac{\mathrm{i} \mathrm{d} \theta}{R \mathrm{e}^{\mathrm{i} \theta}-z} .
$$

Then, rewriting it in terms of $u=\mathrm{e}^{\mathrm{i} \theta}$ we get contour integral along the unit circle

$$
\frac{1}{2 \pi \mathrm{i}} \frac{1-q^{n}}{2} \oint_{|u|=1} \frac{\mathrm{d} u}{u(R u-z)} .
$$

By the residues theorem this integral vanishes

$$
\frac{1-q^{n}}{2 R}\left[\frac{R}{-z}+\frac{R}{z}\right]=0 .
$$

As a result for the line integral we obtain

$$
\mathrm{LI}=\frac{1+q^{n}}{2} z \text {. }
$$

(b) The double integral part in polar coordinates $\zeta=\xi+\mathrm{i} \eta=r \mathrm{e}^{\mathrm{i} \theta}$ is

$$
-\frac{1}{\pi} \iint_{G} \frac{1-q^{n}}{2} \frac{\mathrm{d} \xi \mathrm{d} \eta}{\zeta-z}=-\frac{1-q^{n}}{2 \pi} \int_{0}^{2 \pi} \int_{0}^{R} \frac{r \mathrm{~d} r \mathrm{~d} \theta}{r \mathrm{e}^{\mathrm{i} \theta}-z} .
$$

By substitution $u=\mathrm{e}^{\mathrm{i} \theta}$ we rewrite the angle part of integral as the contour integral along the unit circle $|u|=1$

$$
-\frac{1}{\pi} \iint_{G} \frac{1-q^{n}}{2} \frac{\mathrm{d} \xi \mathrm{d} \eta}{\zeta-z}=-\frac{1-q^{n}}{2 \pi \mathrm{i}} \int_{0}^{R} \mathrm{~d} r \oint_{|u|=1} \frac{\mathrm{d} u}{u(u-z / r)} .
$$

By the residues theorem the contour integral for $|z|>r$ is

$$
\oint_{|u|=1} \frac{\mathrm{d} u}{u(u-z / r)}=2 \pi \mathrm{i}\left(-\frac{r}{z}\right)
$$

and for $|z|<r$ it vanishes. Thus the double integral for $r>|z|$ also vanishes so that the range of integration in $r$ is going from 0 to $|z|$,

$$
\frac{1-q^{n}}{z} \int_{0}^{|z|} r \mathrm{~d} r=\frac{1-q^{n}}{z} \frac{|z|^{2}}{2}
$$

Then finally for the double integral we get

$$
\mathrm{DI}=\frac{1-q^{n}}{2} \bar{z}
$$

Adding the line and the double integrals (A.8), (A.13) together we obtain desired formula (A.2): $\mathrm{LI}+\mathrm{DI}=\frac{1+q^{n}}{2} z+\frac{1-q^{n}}{2} \bar{z}$.

\section{A.2. Generalized analytic function}

For $\Phi_{n}(z)$ in (A.2) as a generalized analytic function, we have the D-bar equation

$$
\frac{\partial \Phi_{n}}{\partial \bar{z}}=\frac{\left(1-q^{n}\right)}{\left(1+q^{n}\right) z+\left(1-q^{n}\right) \bar{z}} \Phi_{n}(z)=A_{n}(z, \bar{z}) \Phi_{n}(z)
$$

where

$$
A_{n}(z, \bar{z})=\frac{\left(1-q^{n}\right)}{\left(1+q^{n}\right) z+\left(1-q^{n}\right) \bar{z}} .
$$

Representation (44) for this function is

$$
\Phi_{n}(z, \bar{z})=\omega(z) \mathrm{e}^{\frac{1}{2 \pi \mathrm{i}} \iint_{D} \frac{A_{n}(\zeta, \bar{\zeta})}{\zeta-z} \mathrm{~d} \zeta \wedge \mathrm{d} \bar{\zeta}} .
$$


To check it we are going to calculate this integral explicitly and find holomorphic function $\omega(z)$ for the disc of radius $R$.

The double integral in exponential is

$I=\frac{1}{2 \pi \mathrm{i}} \iint_{D} \frac{A_{n}(\zeta, \bar{\zeta})}{\zeta-z} \mathrm{~d} \zeta \wedge \mathrm{d} \bar{\zeta}=-\frac{1-q^{n}}{\pi} \iint_{D} \frac{\mathrm{d} \xi \mathrm{d} \eta}{\left[\left(1+q^{n}\right) \zeta+\left(1-q^{n}\right) \bar{\zeta}\right][\zeta-z]}$

where $\zeta=\xi+\mathrm{i} \eta$ and $D=\{\zeta:|\zeta| \leqslant R\}$ or in the polar coordinates $\zeta=r \mathrm{e}^{\mathrm{i} \theta}$,

$I=\frac{q^{n}-1}{\pi} \int_{0}^{R} \int_{0}^{2 \pi} \frac{\mathrm{d} r \mathrm{~d} \theta}{\left[\left(1+q^{n}\right) \mathrm{e}^{\mathrm{i} \theta}+\left(1-q^{n}\right) \mathrm{e}^{-\mathrm{i} \theta}\right]\left[r \mathrm{e}^{\mathrm{i} \theta}-z\right]}=\frac{q^{n}-1}{\pi} \int_{0}^{R} \frac{\mathrm{d} r}{r} I_{0}$

where by complex substitution $u=\mathrm{e}^{\mathrm{i} \theta}$ we have contour integral around unit circle

$$
I_{0}=\frac{1}{\mathrm{i}} \oint_{|u|=1} \frac{\mathrm{d} u}{u} \frac{1}{\left[\left(1+q^{n}\right) u+\left(1-q^{n}\right) \frac{1}{u}\right]\left[u-\frac{z}{r}\right]},
$$

or

$$
I_{0}=\frac{1}{\mathrm{i}\left(1+q^{n}\right)} \oint_{|u|=1} \frac{\mathrm{d} u}{\left[u^{2}+\frac{1-q^{n}}{1+q^{n}}\right]\left[u-\frac{z}{r}\right]} .
$$

For the base $0<q<1$ the integrand has two simple poles inside of the unit circle at $u= \pm \mathrm{i} \sqrt{\frac{1-q^{n}}{1+q^{n}}}$ and for $|z|<r$, one more simple pole at $u=z / r$.

Then by the residues theorem

$$
I_{0}=\frac{2 \pi}{1+q^{n}} \begin{cases}-\frac{1}{\frac{1-q^{n}}{1+q^{n}}+\frac{z^{2}}{r^{2}}}, & |z|>r \\ 0, & |z|<r\end{cases}
$$

Substituting to integral $I$ we get

$$
I=2 \frac{1-q^{n}}{1+q^{n}} \int_{0}^{R} \frac{\mathrm{d} r}{r} \begin{cases}-\frac{1}{\frac{1-q^{n}}{1+q^{n}}+\frac{z^{2}}{r^{2}}}, & |z|>r \\ 0, & |z|<r\end{cases}
$$

or

$$
I=2 \frac{1-q^{n}}{1+q^{n}} \int_{0}^{z} \frac{\mathrm{d} r}{r} \frac{1}{\frac{1-q^{n}}{1+q^{n}}+\frac{z^{2}}{r^{2}}}
$$

By elementary integration

$$
I=\left.\ln \left(r^{2}+\frac{1-q^{n}}{1+q^{n}} z^{2}\right)\right|_{0} ^{z}=\ln \frac{\left(1+q^{n}\right) z+\left(1-q^{n}\right) \bar{z}}{\left(1+q^{n}\right) z}
$$

and for (A.15) then we find

$$
\Phi_{n}(z, \bar{z})=\omega(z) e^{I}=\frac{1+q^{n}}{2} z+\frac{1-q^{n}}{2} \bar{z},
$$

where the analytic function

$$
\omega(z)=\frac{1+q^{n}}{2} z .
$$




\section{A.3. Complex q-binomial as generalized analytic function}

The above results can be applied now for the complex $q$-binomial degree $n$,

$$
(x+\mathrm{i} y)_{q}^{n}=(x+\mathrm{i} y)(x+\mathrm{i} q y) \ldots\left(x+\mathrm{i} q^{n-1} y\right) .
$$

Denoting

$$
\Phi(z)=\Phi_{0}(z) \Phi_{1}(z) \ldots \Phi_{n-1}(z)
$$

where $\Phi_{n}(z)=x+\mathrm{i} q^{n} y=\frac{1+q^{n}}{2} z+\frac{1-q^{n}}{2} \bar{z}$, we have

$$
\frac{\partial}{\partial \bar{z}} \Phi(z, \bar{z})=\Phi(z, \bar{z})(1-q) \sum_{k=1}^{n-1} \frac{[k]_{q}}{\left(1+q^{k}\right) z+\left(1-q^{k}\right) \bar{z}}=A(z, \bar{z}) \Phi(z, \bar{z}),
$$

where

$$
A(z, \bar{z})=(1-q) \sum_{k=1}^{n-1} \frac{[k]_{q}}{\left(1+q^{k}\right) z+\left(1-q^{k}\right) \bar{z}}=\sum_{k=1}^{n-1} A_{n}(z, \bar{z}) .
$$

By the above calculations for the double integral in a disc of radius $R,(\zeta=\xi+\mathrm{i} \eta)$, we obtain

$$
\begin{aligned}
\frac{1}{2 \pi \mathrm{i}} \iint_{D} \frac{A(\zeta, \bar{\zeta})}{\zeta-z} \mathrm{~d} \zeta \wedge \mathrm{d} \bar{\zeta} & =\frac{1}{\pi} \sum_{k=1}^{n-1}\left(q^{k}-1\right) \iint_{D} \frac{\mathrm{d} \xi \mathrm{d} \eta}{\left(\left(1+q^{k}\right) \zeta+\left(1-q^{k}\right) \bar{\zeta}\right)(\zeta-z)} \\
& =\sum_{k=1}^{n-1} \ln \frac{\left(1+q^{k}\right) z+\left(1-q^{k}\right) \bar{z}}{\left(1+q^{k}\right) z}
\end{aligned}
$$

Then

$$
\begin{aligned}
\Phi(z, \bar{z})=(x+\mathrm{i} y)_{q}^{n} & =\omega(z) \exp \left(\frac{1}{2 \pi \mathrm{i}} \iint_{D} \frac{A(\zeta, \bar{\zeta})}{\zeta-z} \mathrm{~d} \zeta \wedge \mathrm{d} \bar{\zeta}\right) \\
& =\omega(z) \exp \left(\ln \prod_{k=1}^{n-1}\left(\frac{\frac{1+q^{k}}{2} z+\frac{1-q^{k}}{2} \bar{z}}{\frac{1+q^{k}}{2} z}\right)\right) \\
& =\omega(z) \prod_{k=1}^{n-1} \frac{\frac{1+q^{k}}{2} z+\frac{1-q^{k}}{2} \bar{z}}{\frac{1+q^{k}}{2} z} \\
& =\omega(z) \prod_{k=1}^{n-1} \frac{2\left(x+\mathrm{i} q^{k} y\right)}{\left(1+q^{k}\right) z}
\end{aligned}
$$

or

$$
(x+\mathrm{i} y)_{q}^{n}=\omega(z) \frac{2^{n}}{z^{n} \prod_{k=0}^{n-1}\left(1+q^{n}\right)}(x+\mathrm{i} y)_{q}^{n} .
$$

As a result we find the next form for the analytic function

$$
\omega(z)=\left(\frac{z}{2}\right)^{n} \prod_{k=0}^{n-1}\left(1+q^{k}\right)
$$




\section{References}

[1] Markushevich A I 1981 Theory of analytic functions Mathematics of XIX Century ed A N Kolmogorov and A P Yushkevich (Moskow: Nauka) (in Russian)

[2] Kac V and Cheung P 2002 Quantum Calculus (New York: Springer)

[3] Pashaev O K and Yilmaz O 2008 Vortex images and $q$-elementary functions J. Phys. A: Math. Theor. 41135207

[4] Pashaev O K 2012 Vortex images, $q$-calculus and entangled coherent states J. Phys.: Conf. Ser. 343012093

[5] Pashaev O K 2014 Two-circles theorem, $q$-periodic functions and entangled qubit states J. Phys.: Conf. Ser. at press

[6] Pashaev O K 2003 Analyticity and integrability as symbolic forms Logic, Mathematics and Philosophy: Proc. National Symp. Mantik, Matematik ve Felsefe (26-28 Sept.) (Istanbul: Istanbul Kultur University Publications)

[7] Jackson F H 1909 A $q$-form of Taylor's theorem Messenger Math. 38 62-4

[8] Hahn W 1949 Beitrage zur theorie der Heineschen Reihen Math. Nachr. 2 340-79

[9] Ernst T 2007 q-complex numbers, a natural consequence of umbral calculus U.U.D.M. Report 2007:44 (Uppsala University)

[10] Ernst T 2008 Motivation for introducing $q$-complex numbers Adv. Dyn. Syst. Appl. 3 107-29

[11] Ernst T 2003 A method for $q$-calculus J. Nonlinear Math. Phys. 10 487-525

[12] Nalci S and Pashaev OK $2010 q$-analog of shock soliton solution J. Phys. A: Math. Theor. $\mathbf{4 3} 445205$

[13] Pashaev O K and Nalci S 2012 -shock soliton evolution Chaos Solitons Fractals 45 1246-54

[14] Vekua I N 1962 Generalized Analytic Functions (Oxford: Pergamon)

[15] Theodoresco N 1936 La derivee areolaire Ann. Roum. Math. 3 3-62

[16] Ablowitz M J and Fokas A S 1997 Complex Variables: Introduction and Applications (Cambridge: Cambridge University Press)

[17] Vitiello G 2012 Fractals, coherent states and self-similarity induced noncommutative geometry arXiv:1206.1854

[18] Vitiello G 2009 Coherent states, fractals and brain waves New Math. Natural Comput. 5 245-64

[19] Vitiello G 2008 Topological defects, fractals and the structure of quantum field theory arXiv:0807.2164

[20] Erzan A 1997 Finite $q$-differences and the discrete renormalization group Phys. Lett. A 225235

[21] Erzan A and Eckmann J P 1997 q-Analysis on fractal sets Phys. Rev. Lett. 783245

[22] Erzan A and Gorbon A $1999 q$-calculus and irreversible dynamics on a hierarchical lattice Turk. J. Phys. 23 9-19

[23] Olemskoi A I 2000 Gauge theory of self-similar system arXiv:cond-mat/0003469v1

[24] Olemskoi A I, Borysov S S and Shuda I A 2010 Statistical field theories deformed within different calculi arXiv:1004.2629v1

[25] Protogenov A P 1994 Quantum group random walks in strongly correlated 2+1 D spin systems ICTP Report IC/94/151 (Trieste: International Centre for Theoretical Physics)

[26] Olemskoi A, Shuda I and Borisyuk V 2010 Generalization of multifractal theory within quantum calculus Europhys. Lett. 8950007

[27] Perelomov A M 1986 Generalized Coherent States and Their Applications (Berlin: Springer)

[28] Shafei Deh Abad A and Milani V 1994 Q-analytic functions on quantum spaces J. Math. Phys. 355074 